\title{
A COMPARATIVE STUDY OF NON-ALCOHOLIC FATTY LIVER DISEASE IN RURAL AND URBAN POPULATION WITH TYPE 2 DIABETES MELLITUS
}

\author{
Davendra Kumar'1, Nikhil Gupta², Jalees Fatima33, Ajay Kumar Mishra4 , Ahraz Ahmad Khan ${ }^{5}$
}

${ }^{1}$ Associate Professor, Department of Medicine, Era's Lucknow Medical College, Lucknow, Uttar Pradesh. ${ }^{2}$ Assistant Professor, Department of Medicine, Era's Lucknow Medical College, Lucknow, Uttar Pradesh. 3 Professor, Department of Medicine, Era's Lucknow Medical College, Lucknow, Uttar Pradesh.

${ }_{4}^{4}$ Associate Professor, Department of Medicine, Era's Lucknow Medical College, Lucknow, Uttar Pradesh. 5Senior Resident, Department of Medicine, Era's Lucknow Medical College, Lucknow, Uttar Pradesh.

ABSTRACT
BACKGROUND
Non-Alcoholic Fatty Liver Disease (NAFLD) is a common liver disease worldwide. The proportion of NAFLD is also higher in
patients with type 2 diabetes than in the general population; 70-75\% patients with type 2 diabetes may have some form of NAFLD.
The objective of this research is to know the prevalence of NAFLD in urban and rural population in our district and to compare
demographic profile, anthropometric measurements and lipid profile in the study group.

\section{METHODS}

This cross-sectional study was performed for 18 months in our institution. Study population included consecutive 160 patients with type 2 diabetes attending the OPD and indoor patients of medicine department. The study group ( $\mathrm{n}=160$ ) was divided into a NAFLD rural population (group $I, n=80$ ) and NAFLD urban population (group II, $n=80$ ). A detailed history regarding the demographic details, physical activity, diet and personal habits were obtained from the patients. After assessment of anthropometric parameters, these patients were subjected to laboratory investigations and ultrasonography. Ultrasonography was used for the detection and gradation of NAFLD according to the standard criteria accepted by the American Gastroenterology Association.

\section{RESULTS}

A total of 160 patients were enrolled during the study. The prevalence of NAFLD was higher in urban patients (58.75\%) than the rural patients (31.25\%). Males were affected more than the female patients in both the groups. Urban population, patients exhibited higher weight, waist circumference, hip circumference, BMI and had earlier age of presentation of NAFLD than the rural population. Both the groups showed high prevalence of metabolic syndrome.

\section{CONCLUSIONS}

The study revealed higher prevalence of NAFLD in urban population as compared to the rural population with males affected more than the female patients. Although the risk factors for NAFLD were similar in both the study groups, better anthropometric parameters (lower weight, waist circumference, hip circumference and BMI had a role in reduced prevalence of NAFLD in rural as compared to Urban population patients.

\section{KEYWORDS}

Non-Alcoholic Fatty Liver Disease, Type 2 Diabetes Mellitus.

HOW TO CITE THIS ARTICLE: Kumar D, Gupta N, Fatima J, et al. A comparative study of non-alcoholic fatty liver disease in rural and urban population with type 2 diabetes mellitus. J. Evolution Med. Dent. Sci. 2016;5(45):2805-2808, DOI: 10.14260/jemds/2016/655

\section{INTRODUCTION}

Non-Alcoholic Fatty Liver Disease (NAFLD) is a common liver disease worldwide. Its reported prevalence varies depending on the population of the study. Changing lifestyle and dietary habits in addition to genetic predisposition has increased the prevalence of obesity and diabetes mellitus and their consequences including NAFLD, specifically in Asian populations. The proportion of NAFLD is also higher in patients with type 2 diabetes than in the general population.[1]; $70-75 \%$ patients with type 2 diabetes may have some form of

Financial or Other, Competing Interest: None.

Submission 05-05-2016, Peer Review 18-05-2016,

Acceptance 20-05-2016, Published 04-06-2016.

Corresponding Author:

Dr. Davendra Kumar

Associate Professor,

Department of Medicine,

Era's Lucknow Medical College,

Lucknow,

Uttar Pradesh.

E-mail: dr.dr_devendra@rediffmail.com

DOI: $10.14260 /$ jemds/2016/655
NAFLD. ${ }^{[2][3]}$ The dependence of NAFLD on obesity is much pronounced in type 2 diabetes mellitus patients, where many workers have found NAFLD to be a universal finding among obese diabetic patients.[4],[5]

In India where nearly $70 \%$ of the population still live in villages and where even primary health care facilities are inadequate, the study of lifestyle disorders in rural population and their prevalence remains rather unexplored. The objective of this research to know the prevalence of NAFLD in urban and rural population in our district and to compare demographic profile, anthropometric measurements and lipid profile in the study group.

\section{MATERIAL AND METHOD}

This cross-sectional study was performed from February 2014 to July 2015 after approval from the Ethics Committee of our Institution. Study population included outdoor patients as well as hospitalized consecutive 160 patients ( 80 Urban and 80 Rural) in the medicine department with type 2 diabetes diagnosed according to the American Diabetes Association (ADA) 2011 criteria. Patients with history of chronic liver disease of any aetiology, space occupying lesion of liver, 
alcohol consumption $>20 \mathrm{~g} /$ day and drugs intake like Tamoxifen, Corticosteroids, Amiodarone, Oestrogen were excluded from the study. The study group was divided into rural population (Group I) and urban population (Group II). A detailed history regarding the demographic details, physical activity, diet and personal habits were obtained from the patients. After assessment of anthropometric parameters, these patients were subjected to laboratory investigations and ultrasonography (Ultrasound machine LOGIQ 5 Pro of GE with 3.5 $\mathrm{MHz}$ convex and $11 \mathrm{MHz}$ linear probe).

Subjects were considered as cases if they have fatty liver according to the standard criteria accepted by the American Gastroenterology Association. NAFLD Grade I - Minimal diffuse increase in the fine echoes. Liver appears bright compared to the cortex of the kidney and normal visualization of diaphragm and intrahepatic vessel borders, NAFLD Grade II - Moderate diffuse increase in the fine echoes. Slightly impaired visualization of the intrahepatic vessels and diaphragm, NAFLD Grade III - Marked increase in the fine echoes. Poor or no visualization of intrahepatic vessels and diaphragm and poor penetration of the posterior segment of the right lobe of the liver.[6] Metabolic syndrome in the study group was detected according to International Diabetes Federation (IDF) Criteria.[7]

\section{Statistical Analysis}

The statistical analysis was done using SPSS (Statistical Package for Social Sciences) Version 15.0 statistical analysis software. The values were represented in Number (\%) and Mean \pm SD. T-test was used to compare continuous variable. The Chi-square test was used to compare categorical variables. A result was deemed statistically significant when $\mathrm{p}<0.05$.

\section{RESULTS}

A total of 160 patients were enrolled during the study. The study group $(n=160)$ was divided into a NAFLD rural population (group $\mathrm{I}, \mathrm{n}=80$ ) and NAFLD urban population (group II, $\mathrm{n}=80)$. NAFLD was detected in 25 (31.25\%) patients in Group I and $47(58.75 \%)$ patients in Group II. (Table 1) There were 17 (68\%) male and $8(32 \%)$ females in NAFLD group I and 25 (53.19\%) males and 22 (46.81\%) female patients in NAFLD group II. The mean age of subjects in NAFLD group I was $54.68+11.65$ years and that of NAFLD group II patients was $47.79+8.92$ years. Mean duration of diabetes mellitus in group I and group II was $76.60+56.79$ months and $50.83+52.96$ months respectively.

Anthropometric variables in patients with NAFLD in both groups are shown in Table 2. Urban population with NAFLD exhibited higher average weight $(71.33 \pm 7.40 \mathrm{kgs}$ vs $67.52 \pm 8.08 \mathrm{kgs}, \mathrm{p}=0.048)$, waist circumference $(90.96 \pm 4.54$ $\mathrm{cm}$ vs. $87.60 \pm 6.92 \mathrm{~cm}, \mathrm{p}=0.016)$, hip circumference $(93.66 \pm 4.66 \mathrm{~cm}$ vs. $90.56 \pm 7.63 \mathrm{~cm}, \mathrm{p}=0.036)$ and Body Mass Index (BMI) $(26.04 \pm 2.35$ vs. $24.40 \pm 2.95, \quad \mathrm{p}=0.012)$ as compared to the rural population with NAFLD.

The serum triglyceride level in both the groups was above normal values. However, comparison of other biochemical parameters including fasting sugar, post-prandial blood sugar, glycosylated haemoglobin, fasting insulin and HOMA-IR and lipid profile between the two NAFLD groups was not significant (Table 3).

In both the study groups, most of the patients were nonvegetarians, group I (96\%) and group II (80.85\%) respectively. Metabolic syndrome was detected higher in group II $47(95.7 \%)$ as compared to the group I patients 21 (84.0\%).

\begin{tabular}{|c|c|c|c|c|c|c|c|}
\hline & \multirow[t]{2}{*}{ Total Subjects } & \multicolumn{2}{|c|}{$\begin{array}{c}\text { Group I }(n=80) \\
\text { Rural }\end{array}$} & \multicolumn{2}{|c|}{$\begin{array}{c}\text { Group II }(n=80) \\
\text { Urban }\end{array}$} & \multicolumn{2}{|c|}{ Statistical Significance } \\
\hline & & No. & $\%$ & No. & $\%$ & $\chi^{2}$ & $\mathbf{P}$ \\
\hline Non-NAFLD & 88 & 55 & 68.75 & 33 & 41.25 & \multirow[t]{2}{*}{12.222} & \multirow[t]{2}{*}{$<0.001$} \\
\hline NAFLD & 72 & 25 & 31.25 & 47 & 58.75 & & \\
\hline
\end{tabular}

\begin{tabular}{|c|c|c|c|c|c|c|}
\hline \multirow{2}{*}{ Anthropometric Variables } & \multicolumn{2}{|c|}{ Group I (Rural NAFLD) } & \multicolumn{2}{c|}{ Group II (Urban NAFLD) } & \multicolumn{2}{c|}{ Statistical Significance } \\
\cline { 2 - 7 } & Mean & SD & Mean & SD & 't' & 'p' \\
\hline Weight (kg) & 67.52 & 8.06 & 71.33 & 7.40 & 2.016 & 0.048 \\
\hline Height (cm) & 166.60 & 7.66 & 165.64 & 6.16 & 0.579 & 0.564 \\
\hline Waist Circumference (cm) & 87.60 & 6.92 & 90.96 & 4.54 & 2.476 & 0.016 \\
\hline Hip Circumference (cm) & 90.56 & 7.63 & 93.66 & 4.66 & 2.140 & 0.036 \\
\hline Waist Hip Ratio & 0.97 & 0.02 & 0.97 & 0.01 & 0.101 & 0.120 \\
\hline BMI & 24.40 & 2.95 & 26.04 & 2.35 & 2.570 & 0.012 \\
\hline
\end{tabular}

\begin{tabular}{|c|c|c|c|c|c|c|}
\hline \multirow{2}{*}{ Parameters } & \multicolumn{2}{|c|}{ Group I } & \multicolumn{2}{|c|}{ Group II } & \multicolumn{2}{|c|}{ Statistical Significance } \\
\hline & Mean & SD & Mean & SD & 't' & 'p' \\
\hline Fasting blood sugar & 193.28 & 47.25 & 185.21 & 43.63 & 0.726 & 0.470 \\
\hline PP & 273.00 & 64.63 & 264.96 & 67.20 & 0.490 & 0.626 \\
\hline HbA1c & 7.76 & 0.59 & 7.65 & 0.71 & 0.669 & 0.506 \\
\hline Serum Cholesterol (mg/dL) & 174.28 & 71.10 & 165.70 & 39.86 & 0.658 & 0.513 \\
\hline Serum Triglycerides (mg/dL) & 204.24 & 116.08 & 190.46 & 76.48 & 0.605 & 0.547 \\
\hline Serum HDL $(\mathrm{mg} / \mathrm{dL})$ & 42.96 & 10.19 & 43.78 & 9.39 & -0.341 & 0.734 \\
\hline Serum LDL (mg/dL) & 90.47 & 46.24 & 84.24 & 28.44 & 0.708 & 0.481 \\
\hline Serum VLDL (mg/dL) & 40.85 & 23.22 & 38.09 & 15.30 & 0.605 & 0.547 \\
\hline Fasting Insulin & 8.28 & 3.39 & 9.43 & 4.01 & -1.212 & 0.230 \\
\hline HOMA-IR & 3.84 & 1.47 & 4.08 & 1.37 & -0.680 & 0.499 \\
\hline
\end{tabular}




\section{DISCUSSION}

The overall prevalence of NAFLD is 15 to $40 \%$ in western countries, while in India the prevalence of NAFLD in the general population varies from $10 \%$ to $30 \%$, the lowest figures being from rural areas of West Bengal and the highest from urban population of Chennai.[8],[9] During the last few decades, a spectrum of research work has shown that diabetics are at a higher risk of non-alcoholic fatty liver disease, which is complicated by the presence of other risk factors such as obesity and metabolic syndrome.[10],[11],,[12],[13],[14],,[15],[16],,[17] Kalra et al in a large multicentric study reported NAFLD in $56.5 \%$ type 2 DM patients, the prevalence of the disease was found to be higher in females $(60 \%)$ as compared to males $(54.3 \%)$ and it increased with increasing age. NAFLD varies from $44.1 \%$ in western India to $72.4 \%$ in northern states.[18]

Our study revealed higher prevalence of NAFLD in the urban group (58.75\%) than the rural population group (31.25\%). Mean age of the rural NAFLD group was significantly higher than urban NAFLD group, thus indicating a probable delaying of NAFLD onset in rural as compared to urban diabetic patients. Although, NAFLD was detected more in males in comparison to females in both groups, no significant association between gender and NAFLD prevalence could be seen. One of the reasons for higher prevalence of males in rural group could be the gender-biased difference in health seeking behaviour in a male dominated society like ours.

We observed that NAFLD patients in the urban population exhibited higher weight, waist circumference, hip circumference and BMI than the rural population. The relationship between anthropometric parameters and NAFLD is well established; however, variable impact of different anthropometric parameters has been shown in different studies. Anthropometric parameters such as BMI and waist/hip ratio have been seen to be associated with causation of NAFLD and its outcome.[19],[20],[21] The dependence of NAFLD on anthropometric measurements is much pronounced in type 2 diabetes mellitus patients where many workers have found NAFLD to be a universal finding among obese patients. [5]

The present study also revealed that the prevalence of metabolic syndrome was significantly higher in urban as compared to rural group and in both the groups, metabolic syndrome was significantly associated with NAFLD. This fact re-emphasized and confirmed that instead of a single risk factor a combination of several variables have a synergistic effect on the occurrence of NAFLD. This finding is in agreement with the observations of previous studies to the extent that NAFLD is often considered to be the hepatic component of metabolic syndrome.[16],[22],[23] This single parameter in itself is capable of explaining the difference in prevalence of NAFLD between rural and urban areas and could explain the multifactorial relationship of NAFLD and also lack of empiricity for univariate relationship.

Although, the majority of NAFLD patients in our study was non-vegetarian, there is varied opinion regarding the effect of diet on the prevalence of NAFLD. Choi et al were of the view that a vegetarian diet does not protect against NAFLD.[24] However, a number of other studies were of the view that diet might have a role in the prevalence and treatment of NAFLD.[25],[26] All these studies indicate that the relationship between diet and NAFLD is not empirical. The findings of the present study also supported the viewpoint that impact of diet on the prevalence of NAFLD might vary from one environment to another environment. The finding also highlighted that the risk factors for NAFLD are also dependent on the overall demographic profile and environmental settings.

In conclusion, our study revealed higher prevalence of NAFLD in urban population as compared to the rural population with males affected more than the female patients. Although the risk factors for NAFLD were similar in both the study groups, better anthropometric parameters (lower weight), waist circumference, hip circumference and BMI had a role in reduced prevalence of NAFLD in rural as compared to Urban population patients.

\section{REFERENCES}

1. Vernon G, Baranova A, Younossi ZM. Systematic review: the epidemiology and natural history of non-alcoholic fatty liver disease and non-alcoholic steatohepatitis in adults. Aliment Pharmacol Ther 2011;34(3):274-85.

2. Medina J, Fernandez-Salazar LI, Garcia-Buey L, et al. Approach to the pathogenesis and treatment of nonalcoholic steatohepatitis. Diabetes Care 2004;27(8):2057-66.

3. Leite NC, Salles GF, Araujo AL, et al. Prevalence and associated factors in non-alcoholic fatty liver disease in patients with type 2 diabetes mellitus. Liver Int 2009;29(1):113-9.

4. Larvine JE, Schwimmer JB. Non-alcoholic fatty liver disease in the paediatric population. Clin Liver Disease 2004;8(3):549-58.

5. Goland S, Shimoni S, Zomitzki $\mathrm{T}$, et al. Cardiac abnormalities as a new manifestation of non-alcoholic fatty liver disease: echocardiographic and tissue doppler imaging assessment. J Clin Gastroenterol 2006;40(10):949-55.

6. Saadeh S, Younossi ZM, Remer EM, et al. The utility of radiological imaging in non-alcoholic fatty liver disease. Gastroenterology 2002;123(3):745-50.

7. The IDF consensus worldwide definition of the metabolic syndrome. Last accessed on 2011 June 11. http://www.idf.org/webdata/docs/IDF_Meta_def_final. pdf .

8. Das K, Mukherjee PS, Ghosh A. Non-obese population in a developing country has a high prevalence of nonalcoholic fatty liver and significant liver disease. Hepatology 2010;51(5):1593-602.

9. Mohan V, Farooq S, Deepa M. Prevalence of non-alcoholic fatty liver disease in urban south Indians in relation to different grades of glucose intolerance and metabolic syndrome. Diabetes Res Clin Pract 2009;84(1):84-91.

10. American diabetes association. Standards of medical care in diabetes-2011. Diabetes Care 2011;34(1):S11-61.

11. Younossi ZM, Gramlich T, Matteoni CA, et al. Nonalcoholic fatty liver disease in patients with type 2 diabetes. Clin Gastroenterol Hepatol 2004;2(3):262-5.

12. Jin $\mathrm{HB}, \mathrm{Gu} \mathrm{ZY}, \mathrm{Yu} \mathrm{CH}$, et al. Association of non-alcoholic fatty liver disease with type 2 diabetes: clinical features and independent risk factors in diabetic fatty liver patients. Hepatobiliary Pancreat Dis Int 2005;4(3):38992.

13. Targher G, Bertolini L, Padovani R, et al. Prevalence of non-alcoholic fatty liver disease and its association with cardiovascular disease among type 2 diabetic patients. Diabetes Care 2007;30(5):1212-8. 
14. Zhou J, Jia WP, Bao YQ, et al. Study on prevalence and risk factors of fatty liver of patients with type 2 diabetes. Zhonghua Yi Xue Za Zhi 2007;87(32):2249-52.

15. Prashanth M, Ganesh HK, Vimal MV, et al. Prevalence of non-alcoholic fatty liver disease in patients with type 2 diabetes mellitus. J Assoc Physicians India 2009;57:20510.

16. Kumar R, Rastogi A, Sharma MK, et al. Clinicopathological characteristics and metabolic profiles of non-alcoholic fatty liver disease in Indian patients with normal body mass index: do they differ from obese or overweight nonalcoholic fatty liver disease? Indian J Endocrinol Metab 2013;17(4):665-71.

17. Nigam $P, B$ hatt $S P$, Misra $A$, et al. Non-alcoholic fatty liver disease is closely associated with sub-clinical inflammation: a case-control study on Asian Indians in north India. PLoS ONE 2013;8(1):e49286.

18. Kalra S, Vithalani M, Gulati G, etal. Study of prevalence of non-alcoholic fatty liver disease (NAFLD) in type 2 diabetes patients in India (SPRINT). J Assoc Physicians India 2013;61(7):448-53.

19. Bellentani $S$, Tiribelli $C$. The spectrum of liver disease in the general population: lesson from the dionysos study. J Hepatol 2001;35(4):531-7.

20. Alam A, Ralhan MAM, Sadiya S, et al. Non-alcoholic fatty liver disease and its anthropometric measurement. IBMC J 2011;2(1):14-6.
21. Otgonsuren $M$, Stepanova $M$, Gerber $L$, et al. Anthropometric and clinical factors associated with morality in subjects with non-alcoholic fatty liver disease. Dig Dis Sci 2013;58(4):1132-40.

22. Praveenraj P, Gomes RM, Kumar S, et al. Prevalence and predictors of non-alcoholic fatty liver disease in morbidly obese south Indian patients undergoing bariatric surgery. Obes Surg 2015;25(11):2078-87.

23. Wang Y, Li YY, Nie YQ, et al. Association between metabolic syndrome and the development of nonalcoholic fatty liver disease. Exp Ther Med 2013;6(1):7784.

24. Choi SH, Oh DJ, Kwon KH, et al. A vegetarian diet does not protect against non-alcoholic fatty liver disease (NAFLD): a cross-sectional study between Buddhist priests and the general population. Turk J Gastroenterol 2015;26(4):336-43.

25. Sofi F, Casini A. Mediterranean diet and non-alcoholic fatty liver disease: new therapeutic option around the corner? World Journal of Gastroenterology 2014;20(23):7339-46.

26. Papandreou D, Andreou E. Role of diet on non-alcoholic fatty liver disease: an updated narrative review. World Journal of Hepatology 2015;7(3):575-82. 